\title{
Drug Delivery to Brain Tumors
}

\author{
Jaishri Blakeley, MD
}

\begin{abstract}
A prerequisite for the efficacy of any cancer drug is that it reaches the tumor in therapeutic concentrations. This is difficult to accomplish in most systemic solid tumors because of factors such as variable hypoxia, intratumoral pressure gradients, and abnormal vasculature within the tumors. In brain cancer, the situation is complicated by the blood-brain barrier (BBB) and bloodcerebrospinal fluid barrier, which serve as physical and physiologic obstacles for delivery of drugs to the central nervous sys tem. Many approaches to overcome, circumvent, disrupt, or manipulate the BBB to enhance delivery of drugs to brain tumors have been devised and are in active investi gation. These approaches include high-dose intravenous chemotherapy, intra-arterial drug delivery, local drug delivery via implanted polymers or catheters, BBB dis ruption, and biochemical modulation of drugs.
\end{abstract}

\section{Introduction}

There has been a recent surge in the number of agents avail able for investigation in brain cancers. Many of these agents have been engineered to target critical tumor pathways and have been successful in historically drug-resistant, systemic solid tumors [1]. Despite these advances in drug discovery and the development of targeted therapies, there has been little impact on the overall prognosis of patients with brain cancer. In fact, although patients with systemic cancer are benefit ing from improved long-term control, the incidence of brain metastases appears to be rising. Similarly, agents that show great promise for primary brain cancers in vitro have had lit tle impact on disease in clinical trials [2••]. There are several possible explanations for the disappointing results in brain cancer therapeutics to date. One of the most notable obstacles is the inability to deliver drugs across the blood-brain barrier (BBB). Given the high frequency of brain cancer (primary and secondary) and its devastating effects, there have been sub stantial efforts to identify techniques to improve delivery of drugs to the central nervous system (CNS).

\section{The Blood-Brain Barrier}

The BBB is a physical and physiologic barrier that regu lates the entry of molecules to the brain. This system is highly effective at protecting the CNS from various tox ins and fluctuations in systemic chemical concentrations. It is also effective at excluding therapeutic

Copyright (C) 2008 by Current Medicine Group LLC

Corresponding author Jaishri Blakeley, MD Johns Hopkins University, Cancer Research Building II, Suite 1M16, 1550 Orleans Street, Baltimore, MD 21231, USA. jblakel3@jhmi.edu.

Disclosure

No potential conflict of interest relevant to this article was reported. 
agents such as chemotherapies and antibiotics. The endothelial cells comprising the BBB have tight junctions, no fenestra tions, and very few pinocytotic vesicles. These cells are buttressed by the end feet of astrocytes (covering $90 \%$ of brain capillaries) and a high concentration of pericytes that further support endothelial cell approximation (Fig. 1) [3••]. An extracellular matrix beyond the astrocyte foot processes provides another layer of protection. These secure junctions and multiple layers of structural barriers functionally mean that agents cannot exit brain capillar ies between endothelial cells.

Physiologic resistance to drug transport at the BBB is equally extensive and includes high electrical resis tance and efflux transporters. The electrical resistance is possibly due to a high expression of occludin; however, expression of organic anion and cation transporters along both the luminal and basolateral membranes also likely contributes to charge gradients (Fig. 1) $[3 \bullet \bullet, 4 \bullet$. This effectively limits the transfer of polar or ionic molecules.

Multiple drug efflux receptors are expressed on brain capillary endothelial cells and the cancer cells them selves [5]. The most notable of these is $\mathrm{P}$ glycoprotein (P-gp); however, several other members of the multidrug resistance family are expressed either on the luminal or brain side of the BBB. Collectively, the receptors are part of the adenosine triphosphatebinding cassette $(\mathrm{ABC})$ transporters. These membrane-bound proteins are widely expressed in normal brain tissue and extrude anticancer agents that have passed into the endothelial cell and back into systemic circulation (Table 1). Although present in normal brain, ABC transporters have increased expres sion in brain cancers [5]. In addition, interventions such as radiation therapy and chemotherapy may increase their expression [6]. Despite the abundance of preclinical studies detailing the role of $\mathrm{ABC}$ transporters in restrict ing drugs to the CNS, the clinical contribution of these transporters to the overall modest response of brain can cers to chemotherapy is unclear. In animal models, P-gp inhibitors given with cytotoxic agents that are substrates for P-gp result in increased penetration of chemotherapy and improved tumor response [7•]. Similar results have been demonstrated for other ABC members, including the multidrug resistance protein 1 (MRP1) and the breast cancer resistance protein (BCRP, ABCG2) [8]. In systemic tumors, expression of various $\mathrm{ABC}$ transporters has been associated with reduced response to chemotherapies and poor overall survival [9]. These findings have led to the investigation of efflux transporter inhibitors given in conjunction with cytotoxic therapies. Despite consider able efforts to advance this approach, results have been mixed in systemic cancers and limited by enhanced toxici ties of the chemotherapies [9]. Clinical trials to evaluate $\mathrm{ABC}$ transporter inhibitors in brain cancers have not been undertaken yet, but the role of active drug efflux is increasingly recognized as a limitation that will need to be addressed to enhance delivery of both systemic and local therapies for brain cancers.

In addition to size, water solubility, and charge, a high degree of plasma protein binding and low serum concen trations predict poor penetration across the BBB. Serum concentrations may be low because of systemic toxicities limiting dosing or because of interaction with concomi tant medications. Enzyme-inducing antiepileptic drugs such as phenytoin, carbamazepine, and phenobarbital induce the $\mathrm{P} 450$ hepatic system and may significantly alter chemotherapy metabolism, resulting in subtherapeutic levels [10]. Newer-generation antiepileptic medications do not induce P450, but drug interactions with anticoagulant 
medications, corticosteroids, and other agents may alter chemotherapy levels. These potential drug interactions have not yet been thoroughly investigated.

Although there is extensive infrastructure to exclude mol ecules from the CNS, there are select mechanisms by which molecules can cross the BBB. Low molecular weight, lipidsoluble, and neutral agents may cross the BBB via passive diffusion. However, they are at risk for extrusion via $\mathrm{ABC}$ proteins such as $\mathrm{P}$-gp and may have rapid transit through the CNS, limiting their efficacy [3••]. Novel approaches for delivery to brain tumors have taken advantage of the three other routes of transport into the CNS that may avoid rapid efflux after entry. These include carrier-mediated transport, receptor-mediated transport/ transcytosis, and absorptive endocytosis (Fig. 1) $[3 \bullet \bullet, 11 \bullet \bullet$.

Carrier-mediated transport is responsible for the movement of small molecules, such as ions and amino acids, required for neuronal health. Carrier receptors are stereospecific and small; therefore, only a limited num ber of substances can potentially be delivered via this technique $[11 \bullet \bullet]$.

Receptor-mediated transport is responsible for the trafficking of larger molecules such as insulin and transferrin $[3 \cdot \bullet, 12]$. In this system, a molecule binds a receptor expressed on the endothelial cell. When there is a "match," it is endocytosed into the cell and transported via vesicles to the basolateral membrane, thereby gaining access to the CNS [11••]. Receptormediated transport has been manipulated to allow anticancer agents to "pig gyback" through the BBB with an agent that binds to a target receptor on the BBB endothelium [13•]. Although promising, particularly for delivery of large agents such as antibodies and genes, this technique is limited by trans port systems that have low affinity and capacity, such that basal levels of the endogenous substrate may interfere with binding of engineered ligands [12].

Absorptive endocytosis relies on the interaction of charged particles. This pathway is most active for the distribution of albumin and other plasma proteins into the CNS and appears to be largely dependent on the elec trostatic relationship between cationized proteins and the BBB. It is currently being investigated for conjugated deliv ery of large immunotherapies (including gene therapy) via nanoparticles [14].

\section{Drug Delivery to Brain Cancers}

\section{Systemic delivery}

Currently, most antineoplastic agents for brain cancers are delivered systemically either as intravenous or oral preparations, depending on the bioavailability of the drug. Although some agents have properties that allow access to brain tumors, the limitations outlined previously impair delivery of most chemotherapies. High-dose intravenous chemotherapy has been investigated for several agents but is generally limited by its associated systemic toxicities. Dose intensification has been effective for methotrexate in the treatment of primary CNS lymphoma, achiev ing higher intracranial concentrations and resulting in improved outcome $[15 \cdot, 16]$. Increasing the dose to enhance delivery across the BBB has 
been considered for other agents, such as etoposide and carboplatin, but such attempts remain experimental [17].

\section{Intra-arterial delivery with BBB disruption}

Intra-arterial (IA) administration of a drug is designed to increase the concentration of a drug delivered to a vascular territory by eliminating first passage metabolism. However, for drugs that have rapid transit through the CNS, there may be limited dwell time, resulting in limited efficacy. Thus far, IA administration alone has not shown improve ment in outcome for brain cancer patients $[18,19]$.

Iatrogenic disruption of the BBB (BBBD) prior to administering systemic chemotherapy has had greater success [20•]. Preclinical models have shown that IA chemotherapy paired with BBBD increases drug con centration in brain parenchyma [21]. The most common approach utilizes osmotic BBBD with agents such as mannitol followed by IA chemotherapy.

However, more recently, MRI-guided ultrasound-induced BBBD has been investigated. This allows more focal BBBD and is well tolerated [15•]. Overall, outcomes with BBBD and IA che motherapy have been mixed across tumor types, and the studies are inconclusive owing to small numbers. How ever, there have been some promising results, including in traditionally difficult tumors such as brainstem gliomas [21]. BBBD and concentrated chemotherapy approaches hold the most promise for anticancer agents that are likely to bind to tumor tissue (and hence, not be rapidly effluxed) and for chemosensitive tumors such as primary CNS lymphoma and germ cell tumors $[12,22,23]$. The side effects associated with BBB disruption and IA drug delivery are generally ischemic in nature and likely related to catheterization of the vessel for delivery as well as the irritative effects of the chemotherapy [20]. There may also be overall enhanced neurotoxicity of the chemotherapy delivered. Currently, BBBD with IA is limited to a select number of experienced institutions, as the procedures are complex, expensive, and associated with uncommon but potentially serious complications.

Conventional radiation therapy used to treat primary and metastatic brain cancer also increases BBB permea bility in both preclinical models and in patients [24]. This is a particularly intriguing observation given the demon strated efficacy of dosing temozolomide concurrently with radiation therapy in patients with newly diagnosed glio blastoma multiforme (GBM) versus the modest activity of temozolomide alone in recurrent GBM [25]. Conversely, it is possible that agents that repair the BBB may inad vertently limit drug access to tumor [26]. Others argue that normalization of the vasculature results in enhanced delivery of drug through normalization of pressure gra dients [27••]. If these hypotheses are true, a sequence of therapies that differentially injures and then repairs the BBB may maximize drug delivery (across a disrupted $\mathrm{BBB}$ ) and then maintain drug concentrations within the tumor (by repairing the BBB), allowing for optimal exposure of the tumor to the drug.

\section{Implanted therapies}

Polymer-based drug delivery has been available for patients with malignant gliomas since the US Food and Drug Administration approved Gliadel (MGI Pharma, Bloom ington, MN) 
for recurrent high-grade gliomas in 1996. Gliadel is a polyanhydride biodegradable polymer wafer impregnated with BCNU (carmustine) that is placed in the surgical cavity at the time of tumor debulking and results in improved survival of roughly 2 months in patients with both newly diagnosed and recurrent malignant gliomas [28,29]. It is well tolerated and associated adverse effects, including increased edema, cerebrospinal fluid leaks, and impaired wound healing, are rare. This approach provides local control of disease but is limited by modest distribu tion of BCNU away from the resection cavity [30]. It is also only available to patients with confined disease who can tolerate a gross total resection. Despite these limitations, Gliadel can be used very effectively as part of a multimo dality approach. The combination of Gliadel and systemic therapies such as temozolomide and $O^{6}$ benzylguanine are under investigation [31]. Finally, recent studies suggest that the maximum tolerated dose of BCNU in polymers in patients with recurrent gliomas may in fact be $40 \mathrm{mg}$, rather than the dose of $7.7 \mathrm{mg}$ currently approved and used in clinical practice [32]. Efficacy studies at the higher dose may show even more robust survival results.

Gliadel has recently been used to treat brain metasta ses. Patients with solitary brain metastases from various solid tumors were treated with resection, placement of Gliadel wafers, and whole-brain radiotherapy [33•]. In 25 patients, there were no local recurrences and overall survival was $25 \%$ at 2 years. Gliadel was well tolerated and appeared to control local disease.

Since the approval of Gliadel, several other devices for local delivery have been developed. Phase II trials using gel technology (ReGel; Protherics, London, UK) with paclitaxel for patients with recurrent gliomas are ongoing. The gel conforms to the shape of the resection cavity and releases paclitaxel over 4 to 6 weeks [34]. Another local delivery technique that has shown early promise is placing cispla tin-infused plates into a tumor. Initial results suggest they are well tolerated in patients with newly diagnosed GBM undergoing radiation therapy. The median survival was 14 months compared with 7 months for the control group [35]. All of these approaches are limited by the need for surgical resection and result in a limited area of distribution; hence, they cannot control the full extent of infiltrative brain can cer. Moreover, the regulatory process for such devices is arduous, requiring a new testing and approval process for each combination of delivery technology and drug. None theless, in select patients, local delivery systems provide a direct, well-tolerated, and effective therapy and may serve as the foundation of a successful multimodal approach.

\section{Convection-enhanced delivery}

Convection-enhanced delivery (CED) uses locally placed catheters within and around a tumor to directly infuse anticancer agents under hydrostatic pressure. This is an attractive approach for agents that are too large to cross the BBB or too toxic for systemic administration [36,37•]. It is a particularly attractive approach for conjugated, targeted toxin therapies, antibodies, and even whole cells. Two recent phase III trials using CED for delivery of such agents have recently been completed: the Phase III Ran domized Evaluation of Convection-Enhanced Delivery of IL13-PE38QQR with Survival Endpoint (PRECISE) trial investigating interleukin-13 conjugated to cintredekin besudotox (PE38QQR), and the TransMID (transferrin-CRM107) trial investigating a modified diphtheria toxin (CRM107) 
conjugated to transferrin in patients with recurrent malignant gliomas. The PRECISE trial showed a median survival of 36.4 weeks (compared with 35.3 weeks with Gliadel) [37]. The TransMID trial was recently stopped at the interval analysis to assess the probability of improvement in overall survival versus standard sec ond-line glioma therapies, and final results are pending. Several other antibody-mediated therapies and immuno therapies have been tested with CED delivery. All have had acceptable toxicities but highly variable efficacy.

CED is subject to many of the same limitations of polymer-based therapies, including limited area of dis tribution and the requirement for surgery. In addition, because drug delivery is dependent on high infusion rates, there may be increased risk of neurotoxicity related to ele vated intracranial pressure. Intuitively, a greater number of catheters placed throughout heterogeneous tumors may result in increased delivery; however, this may be techni cally difficult and has not been demonstrated in clinical practice. This is likely because other factors such as rate of efflux from the CNS, proximity to white matter tracks, and patterns of bulk flow influence the delivery, and there fore the efficacy, of the infused agent [12]. Because the major limitation of CED is the area of drug distribution, imaging techniques such as fluorodeoxyglucose-positron emission tomography (FDG-PET), diffusion-weighted imaging (DWI), MRI, and single photo emission com puted tomography (SPECT) are increasingly being used to image agents within the brain after CED.

\section{Infusion into CSF cavities}

In addition to the BBB, there is a blood-CSF (B-CSF) bar rier. The barrier is composed of fenestrated endothelium on the luminal side and tightly joined epithelial cells without fenestration (the ependyma) on the basolateral side [3••]. Multidrug resistance transporters are also expressed along the B-CSF barrier, limiting access of drugs from systemic circulation to the CSF as well as serving to clear drugs entering the CSF from the brain. Given that leptomeningeal disease occurs in as many as 5\% to $15 \%$ of patients with solid tumors, there have been concerted efforts to enhance the delivery of drug to CSF spaces.

There are essentially three approaches to delivering a drug to the CSF spaces: intrathecal, intraventricular, and intracavitary. Intrathecal administration generally refers to the infusion of drug into the subarachnoid space in the lumbar region. This method requires multiple procedures, and drugs may have limited distribution throughout CSF pathways. Intraventricular administration of a drug requires hardware (ie, implanted reservoirs) but likely results in improved volume of distribution of the drug throughout the CSF pathways. Although relatively well tolerated, there are associated toxicities, including arachnoiditis, meningitis, and focal neurologic injury. Moreover, all of these approaches will result in restricted volume of distribution (and potentially increased toxic ity) if there are CSF flow abnormalities [38]. As these abnormalities are relatively common in patients with lep tomeningeal disease, CSF flow studies should be ordered prior to initiating any CSF drug infusion.

Implanted reservoirs can be used to deliver anticancer therapies directly to tumor cysts or cavities. For instance, an ongoing phase II trial of chlorotoxin coupled with the radioisotope ${ }^{131} \mathrm{I}\left({ }^{131} \mathrm{I}\right.$-TM-601) infuses radioactive therapy into the tumor resection cavity 
via an Ommaya reservoir in patients with recurrent malignant glioma [39]. This approach allows for repeat dosing but remains limited because of a restricted volume of distribution.

\section{Molecular engineering}

Technological advances have allowed modification of exist ing agents to make them more lipophilic, with the goal of enhancing delivery across the BBB [40॰]. There are three broad approaches for increasing lipophilicity of an agent: 1) development of lipophilic analogues or prodrugs, 2) attaching the drug to liposomal carrier systems, and 3) incorporating the drug into lipophilic nanoparticles. Lipo philic analogues have thus far been disappointing in the treatment of primary brain tumors because of decreased cytotoxicity of the primary agent, increased serum protein binding limiting entry into brain, and reduced mobil ity within brain parenchyma. Liposomal carrier systems targeted to receptors on the BBB appear promising in pre clinical models, but there are no clinical data yet available [41,42]. Nanoparticles are polymers between 10 and $1000 \mathrm{~nm}$ in size that use liposomal technology to facilitate delivery of all forms of therapies, from small molecules to genes and cells, into the CNS [40•]. Agents that facilitate imag ing can be encapsulated within the particle along with the anticancer therapy, theoretically allowing direct visualiza tion of drug delivery. Although promising, this technology is currently in early preclinical development.

\section{Assessing Drug Delivery to Tumor}

Independent of the approach used to enhance drug deliv ery, it is critical to understand what dose-concentration curve is required for maximal tumor kill, what dose of drug is delivered to the tumor, over what period of time, and to what volume of tissue. Increasingly, determining intratumoral drug concentrations of investigational agents is recognized as an important step in early drug develop ment. Accordingly, multiple techniques to quantify drug delivery to brain tumors in patients are being used.

Patients can be treated with the drug prior to a planned tumor resection and the resected tissue is sampled for drug concentration and markers of drug activity. This approach is desirable for large agents or agents expected to be membrane bound, but it requires that the patient has surgery, provides drug concentration at only one time point, and allows for possible contamination of the samples by peripheral blood.

Microdialysis is an approach that samples the extracel lular fluid in a focal area of brain and can provide detailed pharmacokinetics of systemically or locally administered agents. The small, flexible catheters have semipermeable membranes that allow sampling of drug concentrations within the extracellular fluid. This has been used extensively in pre clinical trials to assess drug pharmacokinetics within a tumor and more recently has been applied to brain cancer patients [43-45]. Reverse microdialysis employs the same technology but uses it to infuse, rather than collect, a sample. It is similar to CED but with lower hydrostatic pressure and lower volume of drug delivery [46]. Although microdialysis is a powerful and readily available tool for in vivo clinical studies of brain pharmacokinetics, it is limited by the need for placement of the catheter into brain tissue, a limited sampling area, and requiring multiple catheters to assess heterogeneous portions of tumors. This technique is currently restricted to measuring substances less than $20 \mathrm{kD}$ in size. 
Brain imaging approaches are being applied with increasing success to measure the delivery of anticancer agents to brain tumors. For example, molecular imag ing of targeted anticancer agents is being investigated to measure the area of distribution and degree of target inhibition for critical tumor pathways using nuclear medi cine techniques [47]. Interestingly, nuclear medicine has also been used to assess the activity of MDR1 and P-gp in cell culture, suggesting that noninvasive monitoring of key BBB transporters in response to various chemothera peutics may be possible [48•]. Standard MRI can also be used to measure delivery of drugs to a tumor, particularly with agents known to shorten T1, such as the radiation-enhancing agent motexafin gadolinium [49]. Moreover, novel MRI sequences that measure cerebral blood volume are increasingly being used to assess tumor vasculature in response to various antiangiogenic therapies [50].

\section{Conclusions}

Access to the CNS is a critical first step to the success of antineoplastic therapies. Multiple technologies have been developed to accomplish this goal, but additional factors such as tumor cell drug uptake, intracellular metabolism, washout into the extracellular fluid or CSF, inherent tumor sensitivity to chemotherapy, and tumor mecha nisms of resistance all contribute to tumor response and should be considered in drug development [12]. The chal lenge of current clinical research efforts is to define the best antineoplastic agent to deliver for each form of brain cancer and the optimal delivery mechanism for that agent, taking into consideration all of the pharmacokinetic prin ciples specific to that cancer and agent. Finally, regardless of the mechanism of delivery, objective assessment of the drug concentration achieved at the site of disease as well as the impact on clinical outcome is required. Increased understanding of the BBB, the B-CSF barrier, and the mechanisms of drug movement within the CNS as well as ever advancing technology are likely to result in further therapeutic gains for brain cancer in the near future.

\section{References and Recommended Reading}

Papers of particular interest, published recently, have been highlighted as:

- Of importance

•- Of major importance

1. Baselga J, Arteaga CL. Critical update and emerging trends in epidermal growth factor receptor targeting in cancer. J Clin Oncol. 2005; 23:2445-2459. [PubMed: 15753456]

2••. Omuro AM, Faivre S, Raymond E. Lessons learned in the development of targeted therapy for malignant gliomas. Mol Cancer Ther. 2007; 6:1909-1919. [PubMed: 17620423]

3. This is an excellent review of recent research efforts to advance small molecular therapies in the treatment of malignant gliomas and the pitfalls encountered in the clinical investigation of such agents.

30. Deeken JF, Loscher W. The blood-brain barrier and cancer: transporters, treatment, and trojan horses. Clin Cancer Res. 2007; 13:1663-1674. [PubMed: 17363519]

5. This is a detailed review of the BBB physiology with focus on the transporters that impact drug delivery and drug efflux.

4•. Colgan OC, Collins NT, Ferguson G, et al. Influence of basolateral condition on the regulation of brain micro vascular endothelial tight junction properties and barrier function. Brain Res. 2008; 1193:84-92. [PubMed: 18177846] 
7. This is an investigation of the properties of the basolateral (brain) side of the BBB, including the expression of occludin, which was associated with increased electrical resistance and decreased solute penetration. The authors also investigated the independent, modulating role of the astrocytic foot processes in culture.

5. Calatozzolo C, Gelati M, Ciusani E, et al. Expression of drug resistance proteins pgp, MRP1, MRP3, MRP5 and GST-pi in human glioma. J Neurooncol. 2005; 74:113-121. [PubMed: 16193381]

6. Szabo D, Keyzer H, Kaiser HE, Molnar J. Reversal of multidrug resistance of tumor cells. Anticancer Res. 2000; 20:4261-4274. [PubMed: 11205256]

7•. Bihorel S, Camenisch G, Lemaire M, Scherrmann JM. Modulation of the brain distribution of imatinib and its metabolites in mice by valspodar, zosuquidar and elacridar. Pharm Res. 2007; 24:1720-1728. [PubMed: 17380257]

11. This article provides proof of principle that inhibiting multiple drug efflux transporters can enhance drug delivery to brain for select agents. However, this is limited to an animal model and it is not clear that multiple transport inhibition is possible in the clinical setting.

8. de Vries NA, Zhao J, Kroon E, et al. P-glycoprotein and breast cancer resistance protein: two dominant transport ers working together in limiting the brain penetration of topotecan. Clin Cancer Res. 2007; 13:6440-6449. [PubMed: 17975156]

9. Szakacs G, Paterson JK, Ludwig JA, et al. Targeting multidrug resistance in cancer. Nat Rev Drug Discov. 2006; 5:219-234. [PubMed: 16518375]

10. Grossman SA, Hochberg F, Fisher J, et al. Increased 9-aminocamptothecin dose requirements in patients on anticonvulsants. NABTT CNS Consortium. The new approaches to brain tumor therapy. Cancer Chemother Pharmacol. 1998; 42:118-126.

$11 \bullet$. Jones AR, Shusta EV. Blood-brain barrier transport of therapeutics via receptor-mediation. Pharm Res. 2007; 24:1759-1771. [PubMed: 17619996]

16. This article is a good summary of the basic principles of targeting endogenous transport systems to move agents across the $\mathrm{BBB}$ and a detailed review of the accomplishments with receptor-mediated transcytosis, in particular, in the past 10 years.

12. Groothuis DR. The blood-brain and blood-tumor barriers: a review of strategies for increasing drug delivery. Neuro Oncol. 2000; 2:45-59. [PubMed: 11302254]

13. Xia CF, Zhang Y, Zhang Y, et al. Intravenous siRNA of brain cancer with receptor targeting and avidin-biotin technology. Pharm Res. 2007; 24:2309-2316. [PubMed: 17926121]

19. This is an in vivo study demonstrating the efficacy of delivery of small interfering RNA to rat glioma cells using the technique of receptor-mediated transport targeting the transferrin receptor.

14. Lu W, Zhang Y, Tan YZ, et al. Cationic albumin-con jugated pegylated nanoparticles as novel drug carrier for brain delivery. J Control Release. 2005; 107:428-448. [PubMed: 16176844]

15•. Muldoon LL, Soussain C, Jahnke K, et al. Chemotherapy delivery issues in central nervous system malignancy: a reality check. J Clin Oncol. 2007; 25:2295-2305. [PubMed: 17538176]

22. This is a detailed review that highlights the theoretical consider ations of drug delivery, the impact of delivery on drug discovery and development for brain cancers, and current approaches to enhance drug delivery to tumor.

16. Mohile NA, Abrey LE. Primary central nervous system lymphoma. Neurol Clin. 2007; 25:11931207. [PubMed: 17964031]

17. Vinolas N, Gil M, Verger E, et al. Pre-irradiation semi-intensive chemotherapy with carboplatin and cyclophosphamide in malignant glioma: a phase II study. Anticancer Drugs. 2002; 13:163167. [PubMed: 11901309]

18. Dropcho EJ, Rosenfeld SS, Vitek J, et al. Phase II study of intracarotid or selective intracerebral infusion of cisplatin for treatment of recurrent anaplastic gliomas. J Neurooncol. 1998; 36:191198. [PubMed: 9525819]

19. Ashby LS, Shapiro WR. Intra-arterial cisplatin plus oral etoposide for the treatment of recurrent malignant glioma: a phase II study. J Neurooncol. 2001; 51:67-86. [PubMed: 11349883]

20•. Fortin D, Desjardins A, Benko A, et al. Enhanced chemo therapy delivery by intraarterial infusion and blood-brain barrier disruption in malignant brain tumors: the Sher brooke experience. Cancer. 2005; 103:2606-2615. [PubMed: 15880378] 
28. This is an informative summary report on the outcomes (survival, progression-free survival, and complications) in various tumor types after therapy with BBB disruption and intra-arterial chemotherapy.

21. Remsen LG, Trail PA, Hellstrom I, et al. Enhanced delivery improves the efficacy of a tumorspecific doxorubicin immunoconjugate in a human brain tumor xenograft model. Neurosurgery. 2000; 46:704-709. [PubMed: 10719867]

22. Kraemer DF, Fortin D, Doolittle ND, Neuwelt EA. Associa tion of total dose intensity of chemotherapy in primary central nervous system lymphoma (human non-acquired immunodeficiency syndrome) and survival. Neurosurgery. 2001; 48:1033-1040. discussion 1040-1041. [PubMed: 11334269]

23. Jahnke K, Kraemer DF, Knight KR, et al. Intraarterial chemotherapy and osmotic blood-brain barrier disruption for patients with embryonal and germ cell tumors of the central nervous system. Cancer. 2008; 112:581-588. [PubMed: 18072268]

24. Cao Y, Tsien CI, Shen Z, et al. Use of magnetic resonance imaging to assess blood-brain/bloodglioma barrier open ing during conformal radiotherapy. J Clin Oncol. 2005; 23:4127-4136. [PubMed: 15961760]

25. van Vulpen M, Kal HB, Taphoorn MJ, El-Sharouni SY. Changes in blood-brain barrier permeability induced by radiotherapy: implications for timing of chemotherapy? [review]. Oncol Rep. 2002; 9:683-688. [PubMed: 12066192]

26. Ma J, Pulfer S, Li S, et al. Pharmacodynamic-mediated reduction of temozolomide tumor concentrations by the angiogenesis inhibitor TNP-470. Cancer Res. 2001; 61:5491-5498. [PubMed: 11454697]

27••. Jain RK. Normalization of tumor vasculature: an emerging concept in antiangiogenic therapy. Science. 2005; 307:58-62. [PubMed: 15637262]

36. This is a concise but comprehensive discussion of solid tumor vas culature and the potential benefits of "normalizing" tumor blood vessels to enhance treatment efficacy. It also examines the potential risks and benefits of antiangiogenic therapies in clinical practice.

28. Brem H, Piantadosi S, Burger PC, et al. Placebo-controlled trial of safety and efficacy of intraoperative controlled delivery by biodegradable polymers of chemotherapy for recurrent gliomas. The polymer-brain tumor treatment group. Lancet. 1995; 345:1008-1012. [PubMed: 7723496]

29. Westphal M, Hilt DC, Bortey E, et al. A phase 3 trial of local chemotherapy with biodegradable carmustine (BCNU) wafers (Gliadel wafers) in patients with primary malignant glioma. Neuro Oncol. 2003; 5:79-88. [PubMed: 12672279]

30. Fung LK, Ewend MG, Sills A, et al. Pharmacokinetics of interstitial delivery of carmustine, 4hydroperoxycy clophosphamide, and paclitaxel from a biodegradable polymer implant in the monkey brain. Cancer Res. 1998; 58:672-684. [PubMed: 9485020]

31. Weingart J, Grossman SA, Carson KA, et al. Phase I trial of polifeprosan 20 with carmustine implant plus continu ous infusion of intravenous O6-benzylguanine in adults with recurrent malignant glioma: new approaches to brain tumor therapy CNS consortium trial. J Clin Oncol. 2007; 25:399-404. [PubMed: 17264335]

32. Olivi A, Grossman SA, Tatter S, et al. New Approaches to Brain Tumor Therapy CNS Consortium: Dose escalation of carmustine in surgically implanted polymers in patients with recurrent malignant glioma: a New Approaches to Brain Tumor Therapy CNS Consortium trial. J Clin Oncol. 2003; 21:1845-1849. [PubMed: 12721262]

33. Ewend MG, Brem S, Gilbert M, et al. Treatment of single brain metastasis with resection, intracavity carmustine polymer wafers, and radiation therapy is safe and provides excellent local control. Clin Cancer Res. 2007; 13:3637-3641. [PubMed: 17575228]

43. This was the first clinical application of local polymer delivery to brain metastases to demonstrate tolerability and local control.

34. Vukelja SJ, Anthony SP, Arseneau JC, et al. Phase 1 study of escalating-dose OncoGel (ReGel/ paclitaxel) depot injec tion, a controlled-release formulation of paclitaxel, for local management of superficial solid tumor lesions. Anticancer Drugs. 2007; 18:283-289. [PubMed: 17264760] 
35. Sheleg SV, Korotkevich EA, Zhavrid EA, et al. Local chemotherapy with cisplatin-depot for glioblastoma multiforme. J Neurooncol. 2002; 60:53-59. [PubMed: 12416546]

36. Patel SJ, Shapiro WR, Laske DW, et al. Safety and feasibility of convection-enhanced delivery of cotara for the treatment of malignant glioma: initial experience in 51 patients. Neurosurgery. 2005; 56:1243-1252. discussion 1252-1253. [PubMed: 15918940]

37•. Kunwar S, Prados MD, Chang SM, et al. Direct intrace rebral delivery of cintredekin besudotox (IL13-PE38QQR) in recurrent malignant glioma: a report by the cintredekin besudotox intraparenchymal study group. J Clin Oncol. 2007; 25:837-844. [PubMed: 17327604]

48. This is a report on data from phase I studies (total number of patients $=51$ ) with IL13-PE38QQR highlighting the importance of catheter position relative to tumor bulk and the adverse events, primarily in the CNS.

38. Grossman SA, Trump DL, Chen DC, et al. Cerebrospinal fluid flow abnormalities in patients with neoplastic meningi tis. An evaluation using 111indium-DTPA ventriculography. Am J Med. 1982; 73:641-647. [PubMed: 6814249]

39. Mamelak AN, Rosenfeld S, Bucholz R, et al. Phase I single-dose study of intracavitaryadministered iodine-131-TM-601 in adults with recurrent high-grade glioma. J Clin Oncol. 2006; 24:3644-3650. [PubMed: 16877732]

40•. Reddy GR, Bhojani MS, McConville P, et al. Vascular targeted nanoparticles for imaging and treatment of brain tumors. Clin Cancer Res. 2006; 12:6677-6686. [PubMed: 17121886]

52. This in vivo study demonstrated the principles of the flexibility of nanoparticle therapy allowing drug delivery, as well as imaging of phar macokinetics by incorporating agents allowing visualization via MRI.

41. Yoshida J, Mizuno M. Clinical gene therapy for brain tumors. Liposomal delivery of anticancer molecule to glioma. J Neurooncol. 2003; 65:261-267.

42. Shao K, Hou Q, Duan W, et al. Intracellular drug delivery by sulfatide-mediated liposomes to gliomas. J Control Release. 2006; 115:150-157. [PubMed: 16963144]

43. de Lange EC, de Vries JD, Zurcher C, et al. The use of intracerebral microdialysis for the determination of phar macokinetic profiles of anticancer drugs in tumor-bearing rat brain. Pharm Res. 1995; 12:1924-1931. [PubMed: 8786967]

44. Olson J, Blakeley J, Grossman SA, et al. Differences in the distribution of methotrexate into high grade gliomas following intravenous administration, as monitored by microdialysis, are associated with blood brain barrier integrity [abstract]. J Clin Oncol. 2006; 24:1548.

45. Portnow J, Badie B, Xi B, et al. Intracerebral microdialysis for determination of the neuropharmacokinetics and neuropharmacodynamics of temozolomide in patients with primary or metastatic brain tumors [abstract]. J Clin Oncol. 2007; 25:2074.

46. Bergenheim AT, Roslin M, Ungerstedt U, et al. Metabolic manipulation of glioblastoma in vivo by retrograde micro dialysis of L-2, 4 diaminobutyric acid (DAB). J Neurooncol. 2006; 80:285-293. [PubMed: 16773220]

47. Mishani E, Abourbeh G. Cancer molecular imaging: radio nuclide-based biomarkers of the epidermal growth factor receptor (EGFR). Curr Top Med Chem. 2007; 7:1755-1772. [PubMed: 17979785]

48•. Sharma V, Prior JL, Belinsky MG, et al. Characterization of a $67 \mathrm{Ga} / 68 \mathrm{Ga}$ radiopharmaceutical for SPECT and PET of MDR1 P-glycoprotein transport activity in vivo: valida tion in multidrugresistant tumors and at the blood-brain barrier. J Nucl Med. 2005; 46:354-364. [PubMed: 15695797]

61. This is an in vitro study demonstrating the ability to assess membrane transport function noninvasively. Although early, the results suggest that in vivo imaging of the BBB to measure degree of disruption and predict the likelihood of transport of various agents at a given time may be possible.

49. Wu GN, Ford JM, Alger JR. MRI measurement of the uptake and retention of motexafin gadolinium in glioblas toma multiforme and uninvolved normal human brain. J Neurooncol. 2006; 77:95-103. [PubMed: 16547607] 
50. Batchelor TT, Sorensen AG, di Tomaso E, et al. AZD2171, a pan-VEGF receptor tyrosine kinase inhibitor, normalizes tumor vasculature and alleviates edema in glioblastoma patients. Cancer Cell. 2007; 11:83-95. [PubMed: 17222792] 


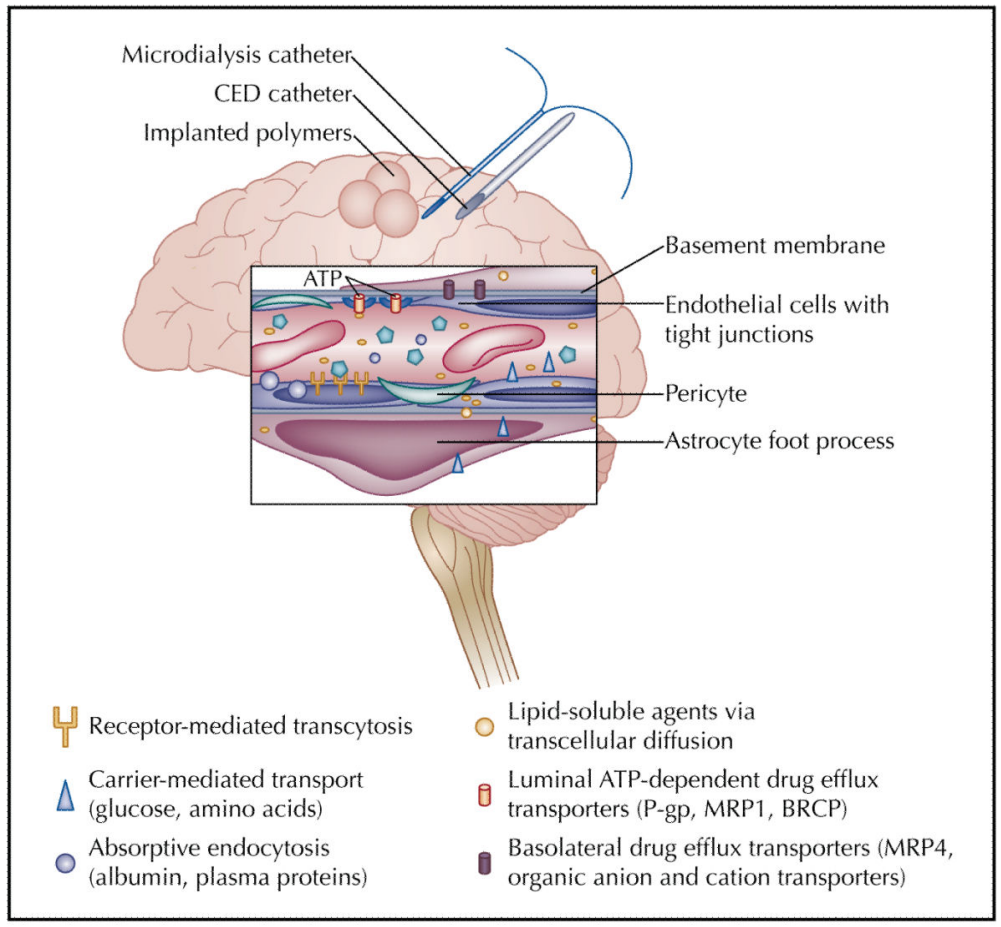

Figure 1.

Schematic of the components of the blood-brain barrier (BBB), includ ing endothelial cells with tight junctions, pericytes, basement membrane, drug efflux transporters, and astrocytic foot processes. Various mechanisms for transport across the BBB, including transcellular diffusion, absorptive endocytosis, carrier-mediated transport, and receptor-mediated transcytosis, are also represented. Finally, approaches to deliver drugs directly to the brain and to assess drug concentration within brain tissue are displayed. ATP—adenosine triphosphate; BCRP—breast cancer resistance protein; CED—convection-enhanced delivery; MRP —multidrug resistance protein; P-gp-P glycoprotein. 


\section{Table 1}

Drug efflux transporters at the blood-brain barrier

\begin{tabular}{lcc}
\hline Transporter & Location & Anticancer agent substrates \\
P glycoprotein & $\begin{array}{c}\text { Luminal of BBB, luminal } \\
\text { of B-CSF barrier }\end{array}$ & $\begin{array}{c}\text { Doxorubicin, epirubicin, daunorubicin, docetaxel, paclitaxel, } \\
\text { vinblastine, vincristine, etoposide, methotrexate }\end{array}$ \\
MRP1 & $\begin{array}{c}\text { Luminal of BBB, basolateral } \\
\text { of B-CSF barrier }\end{array}$ & Similar to P-gp plus teniposide and melphan \\
MRP2 & Luminal & Similar to P-gp \\
MRP3 & Luminal & Similar to P-gp \\
MRP4 & Luminal and basolateral \\
MRP5 & Luminal \\
MRP6 & Luminal \\
$\begin{array}{c}\text { Breast cancer } \\
\text { resistance protein }\end{array}$ & Luminal & Methotrexate, 6-mercaptopurine, thioguanine \\
$\begin{array}{c}\text { Organic anion and } \\
\text { cation transporters }\end{array}$ & $\begin{array}{c}\text { Luminal and basolateral } \\
\text { of BBB, B-CSF barrier }\end{array}$ & 6-mercaptopurine, thioguanine \\
BBB-blood-brain barrier; B-CSF-blood-cerebrospinal fluid barrier; MRP-multidrug resistance protein; P-gp-P glycoprotein.
\end{tabular}

